\title{
Millimole per Liter per Microgram per Kilogram
}

National Cancer Institute

\section{Source}

National Cancer Institute. Millimole per Liter per Microgram per Kilogram. NCI

Thesaurus. Code C119419.

A unit of concentration equal to millimoles per liter, divided by micrograms per kilogram. 